\title{
Comparison Between Accuracy of Two Different Electronic Apex Locators in Determining Working Length of Root Canals: An In Vitro Study
}

\author{
Dr. Pheiroijam Herojit Singh ${ }^{1 *}$, Dr. Ningthoukhongjam Rati Devi², Dr. N. Sukumar \\ Singh $^{3}$ \\ ${ }^{1}$ Post-Graduate Student, Department of Pediatric and Preventive Dentistry, Jaipur Dental College, \\ Rajasthan, India. \\ ${ }^{2}$ Assistant Professor, Department of Conservative Dentistry \& Endodontics, Dental College JNIMS, \\ Imphal, Manipur, India. \\ ${ }^{3}$ Associate Professor, Department of Conservative Dentistry \& Endodontics, Dental College JNIMS, \\ Imphal, Manipur, India.
}

\section{Original Article}

\section{Address for Correspondence Author}

Dr. Pheiroijam Herojit Singh, Post-Graduate Student, Department of Pediatric and Preventive Dentistry, Jaipur Dental College, Rajasthan, India.

E-mail: go4herojit@gmail.com

Crossref doi: https://doi.org/10.36437/ijdrd.2020.2.1.M

\section{ABSTRACT}

Background: Accurate determination of the working length of root canals is an important factor for the success of endodontic therapy.

Aim: Two different apex locators namely Propex Pixi (Dentsply) and Canal Pro (Coltene) are used to determine the working length of root canals and their results are compared to determine their accuracy.

Material and Methods: Twenty extracted permanent single-rooted teeth are used for the study. To determine the working length of root canals of each tooth, two different types of electronic apex locators, Propex Pixi (Dentsply) and CanalPro (Coltene) are used and their results are compared.

Result: There was a significant difference in accuracy between the two apex locators in determining the working length of root canals ( $\mathrm{p}$-value $<0.05$ ). Canal Pro was more accurate than Propex Pixi.

Keywords: Apex Locator, Patients, Root Canals.

\section{Introduction}

The success of root canal treatment depends on a number of factors such as extent of dental caries, chemicobiomechanical preparation, obturation and remaining tooth structure. The extent of preparation up to the cemento-dentinal junction or the apical constriction is considered one of the important factors for success of any root canal treatment. The cemento-dentinal junction is the histological landmark and is clinically difficult to locate it. Therefore the apical constriction is regarded as the clinical landmark up to which preparation and obturation should end for the success of treatment. 
Clinically the apical constriction is traditionally located by the tug back feel of master gutta-percha cone but it is not reliable. The canal can be sclerosed or constricted or secondary curvature or root resorption may give a deceptive result. Therefore the use of modernized equipment or method for location of apical constriction clinically is important. The use of electronic apex locators can be considered an important aspect of clinical practices.

The present in vitro study was conducted to evaluate the accuracy of two apex locators namely Propex Pixi (Dentsply) and Canal Pro (Coltene) in determining the working length of root canals.

\section{Materials and Methods}

\section{Collection and Preparation of Samples}

Twenty extracted permanent single-rooted teeth were collected from the Department of Oral Surgery, Dental College\& Hospital, JNIMS, Imphal, Manipur, India. All the teeth with complete root length and without any sign of resorption were included. The teeth were immersed in a 5.2\% sodium hypochlorite solution for 48 hours. The teeth were then thoroughly scaled with an ultrasonic scaler (Woodpecker) to remove any hard tissue or mucosa attached to the root surface. Access to the root canal of all the teeth was prepared with a sterile round bur (Mani Inc.). A 15 \# NiTi K-file (Waldent) was used to negotiate the canals using alternate sodium hypochlorite and normal saline irrigation.

\section{Measurement of actual working length}

A 15\# NiTi K-file (Waldent) was inserted inside the canal until it became visible at the apical foramen. The rubber stop was adjusted, the file was removed and the distance between the base of rubber stop and file tip was measured using an endo gauge. To obtain the actual working length, $0.5 \mathrm{~mm}$ was deducted from this measured length.

\section{Measurement of working length with electronic apex locators}

A plastic box was used for making a model for the study. Alginate was poured into the box and all the twenty teeth were mounted vertically up to their cemento-enamel junction. Alginate acts as the electro-conductive medium and all measurements were conducted within 1 hour of pouring the alginate.

Two electronic apex locators were selected for the study, Propex Pixi (Dentsply) and CanalPro (Coltene). Each tooth was subjected to each electronic apex locator for the determination of working length measurement. The entire technique was performed and the measurements were recorded by a single operator. Before the start of the operation, precaution was taken to see all the circuits, batteries and the operating modes of all apex locators are fully functional.

All the canals of each tooth were dried using absorbent paper points (Meta BioMed) before the start of operation. A 15\# NiTi K-file (Waldent) was attached to the file clip of the apex locator to be used and introduced inside the canal until the apex locator showed the "0.5 $\mathbf{~ m m " ~ r e a d i n g . ~ T h e ~ r u b b e r ~ s t o p ~ w a s ~}$ adjusted and the file was removed and the distance between the base of rubber stop and file tip was measured using an endo gauge. Measurements were considered as valid if they were stable for at least 5 seconds. The recorded values were tabulated.

\section{Result}

The recorded readings of the two apex locators were compared with the recorded values of actual working length being measured. Statistical analysis of the recorded readings was done by MedCalc's comparison of 
means. Statistical readings were considered significant when $\mathrm{p}<0.05$. A Significant difference was found between readings of Propex Pixi and actual length being measured (Table 1).

\begin{tabular}{|c|l|c|c|}
\hline Group & Mean & Standard Deviation & p-Value \\
\hline Actual Length & 21.32 & 1.84 & ..0444* $^{*}$ \\
\hline Propex Pixi & 20.11 & 1.84 \\
\hline
\end{tabular}

Table1. Comparison of mean working length between actual length and Propex Pixi (in mm) There was no significant difference between recordings of Canal Pro and actual working length being measured (Table 2).

\begin{tabular}{|c|c|c|c|}
\hline Group & Mean & Standard Deviation & p-Value \\
\hline Actual Length & 21.32 & 1.84 & $\mathbf{1 . 0 0 0 0}^{*}$ \\
\hline Canal Pro & 21.35 & 1.81 & \\
\hline \multicolumn{2}{|c|}{${ }^{*}$ p value $>0.05$ (No significant difference) } \\
\hline
\end{tabular}

Table2. Comparison of mean working length between actual length and CanalPro (in mm)

\section{Discussion}

Accurate determination of the working length of root canals is one of the main factors for the success of endodontic treatment. Instrumentation and obturation of root canals should be limited to the apical constriction which is a key factor for successful root canal treatment ${ }^{1}$.

Custer in 1918 was the first to use electric current to determine the working length. In 1962, Sunada reported that there is a constant value of $6.5 \mathrm{k} \Omega$ of electrical resistance between the mucous membrane and the periodontium which is possible to use in the determination of working length. This idea is being utilized as the principle of working of electronic apex locators. Lucena-Martin, et al., conducted a study to evaluate several apex locators and found that Root ZX II was more reliable among them ${ }^{2}$. Haffneret. al, compared apex locators with a microscope and found that apex locators were more reliable in determining working length ${ }^{3}$. Sadeghi and Abolghasemi compared apex locators with the conventional radiographic method in straight and curved root canals and found that apex locators were more reliable in determining working length irrespective of root curvatures 4 . Studies have shown that working length determination with an apex locator is easier, reliable, saves chair side time and no risk of radiation exposure than a conventional radiograph.

In the present study, readings of two electronic apex locators namely Propex Pixi and CanalPro were compared with the readings of the actual working length. The results demonstrated that readings of Canal Pro were found to be more accurate than Propex Pixi.

\section{Conclusion}

The present study was conducted to compare the accuracy of two electronic apex locators in determination of working length in permanent anterior teeth. 


\section{The following points can be concluded from this study:}

1. Working length determination with electronic apex locator is more reliable and accurate method.

2. Minor difference was observed between the readings of apex locators and actual working length.

The use of apex locator in clinical endodontic practices is both accurate and safe to patients. However, care must be taken in patients with implanted devices such as pace-makers, defibrillators, etc.

\section{References}

1. Ebrahim AK, Wadachi R, Suda H. Electronic apex locators - a review. J Med Dent Sci. 2007; 54:125-136.

2. Lucena-Martín C, Robles-Gijón V, Ferrer-Luque CM, de Mondelo JM. In vitro evaluation of the accuracy of three electronic apex locators. J Endod. 2004; 30(4):231-233.

3. Haffner C, Folwaczny M, Galler K, Hickel R. Accuracy of electronic apex locators in comparison to actual length-an in vivo study. J Dent. 2005; 33(8):619-625.

4. Sadeghi S, Abolghasemi M. A comparison between the Raypex5 apex locator and conventional radiography for determining working length of straight and curved canals. Iran Endod J. 2008; 2 (3):101-104.

How to cite this Article: Dr. Pheiroijam Herojit Singh ${ }^{1 *}$, Dr. Ningthoukhongjam Rati Devi², Dr. N. Sukumar Singh ${ }^{3}$, : Comparison Between Accuracy of Two Different Electronic Apex Locators in Determining Working Length of Root Canals: An In Vitro Study

Int. J. Drug Res. Dental Sci., 2020; 2(1):14-17.

Source of Support: Nil, Conflict of Interest: None declared.

Received: 24-1-2020 Revised: 12-2-2020 Accepted: 14-2-2020 